\title{
DESVENDANDO OS PADRÕES DE PREFERÊNCIA DE HABITAT DE LARVAS DE SIMULIIDAE (DIPTERA) NEOTROPICAIS E SUAS IMPLICAÇÕES PARA O CONTROLE DO VETOR
}

\author{
Ivete Ramos de Arruda BUFFOLO ${ }^{1}$, Tainá Maria Miranda SOUZA $^{1}$, Suzana Silva dos SANTOS $^{1}$, Tayanna \\ RODRIGUES $^{1}$, Lidiane Coelho BERBERT $^{1,2}$, Tatiana Nascimento DOCILE ${ }^{1,3}$ \& Ronaldo FIGUEIRÓ ${ }^{1,2,4}$ \\ ${ }^{1}$ Fundação Centro Universitário Estadual da Zona Oeste, Laboratório de Biotecnologia Ambiental, Rio de Janeiro, \\ RJ, Brasil. \\ ${ }^{2}$ Fundação Centro Universitário Estadual da Zona Oeste, Programa de Pós-graduação em Ciência e Tecnologia \\ Ambiental Rio de Janeiro, RJ, Brasil. \\ ${ }^{3}$ Universidade Federal do Rio de Janeiro, Laboratório de Entomologia / Programa de Pós-graduação em Ecologia - \\ Rio de Janeiro, RJ, Brasil. \\ ${ }^{4}$ Centro Universitário de Volta Redonda, Volta Redonda, Rio de Janeiro, Brasil. \\ *Autor para correspondência: ronaldofigueiro@gmail.com
}

http://dx.doi.org/10.18571/acbm.103

\section{RESUMO}

A família Simuliidae (Diptera) é composta por insetos de hábito hematofágico que apresentam distribuição global e são vetores da Oncocercose e da Mansonelose. Estes organismos tem como criadouros de suas formas imaturas ambientes de água corrente, onde suas larvas e pupas se desenvolvem. Devido à larva constituir o elo mais frágil do ciclo destes insetos, os esforços para seu controle devem ser direcionados aos criadouros destas formas, que são córregos e rios. No entanto, um melhor entendimento dos padrões de preferência de habitat destes organismos podem nortear iniciativas de controle menos agressivas ao ambiente e mais eficazes. Esta revisão busca traçar um panorama do estado da arte na literatura do conhecimento dos padrões de distribuição e preferência habitat de larvas de simulídeos neotropicais e de suas perspectivas para o controle.

Palavras-chave: Preferência de habitat; Controle; Simulídeos.

\begin{abstract}
The Simuliidae family (Diptera) is composed by hematophagous insects which show a world wide distributional pattern and are vectors of the Onchocerciasis and the Mansonelosis. These organisms breed in their immature forms in running water environments, where their larvae and pupae develop. Due to the larvae represent the weakest link in the life cycle of these insects, the efforts to their control should be focused to the breeding sites of these forms, which are rivers and streams. However, a better understanding of their habitat preference patterns may guide more efficient and more environment-friendly control initiatives. This review seeks to draw an overview of the state of the art from the literature on the knowledge of distributional and habitat preference patterns of neotropical Black fly larvae, and its perspectives for the control of these insects.
\end{abstract}

Keywords: Habitat preference; Control; Simuliids. 


\section{Introdução}

As doenças transmitidas por vetores constituem, ainda hoje, importante causa de morbidade no Mundo (TAUIL, 2002). Dentre esses vetores, os simulídeos pertencem a uma família de importância médica, por compreender espécies vetoras da Oncocercose (REY, 1991) e da Mansonelose (CERQUEIRA, 1959; SHELLEY et al.,1980; MORAES et al.,1985), e também serem relacionados na literatura com o desenvolvimento da doença autoimune Pênfigo Foliáceo (EATON et al.,1998). Além disso, as picadas de fêmeas causam alergias para os seres humanos e certos animais, causando perdas no turismo e na agricultura (COUCEIRO et al., 2014). Por esses motivos, estes insetos têm sido alvo de grandes esforços para o seu controle nas últimas décadas (ARAÚJO-COUTINHO,1995).

Os borrachudos (Diptera: Simuliidae) são vetores de doenças para os homens e os animais. A picada por um inseto adulto gera extremo desconforto e agravos à saúde. Esta situação é conhecida há muitos anos em especial nas áreas rurais onde sua presença perturba e limita as atividades cotidianas das pessoas e dos moradores, além de causar perdas na produtividade dos animais e prejuízos aos polos turísticos (MARDINI et al., 2006).

Os insetos adultos da família Simuliidae estão sujeitos a parasitemias por vermes nematóides (superfamília Filaroidea e Mermithoidea) (CROSSKEY, 1990). As filárias apresentam especial importância por utilizarem os simulídeos como hospedeiros intermediários, sendo suas larvas transmitidas a hospedeiros definitivos vertebrados no momento em que a fêmea realiza seu repasto sanguíneo (CAMPOS e ANDRADE, 1999). Algumas espécies da família Simuliidae apresentam competência vetorial para transmitir espécies de nematóides dos gêneros Onchocerca, Mansonella, Splendidofilaria e Dirofilaria, motivo pelo qual apresentam grande importância médica e veterinária.

Estudos realizados em outros países constataram que as infestações deste inseto estavam relacionadas com o aumento da deposição da matéria orgânica nos rios, devido ao lançamento de despejos domésticos, agrícolas e industriais (PEDROSO-DE-PAIVA e BRANCO, 2000).

A Oncocercose, também conhecida como cegueira dos rios é ocasionada pela microfilária Onchocerca volvulus Leuckart, 1893 (REY, 1991). Como descrito por Mardini et al.(2002), essa doença causada por uma filária, que se desenvolve no sangue do homem e que produz grandes tumores subcutâneos. Quando o verme se localiza nos olhos, causa cegueira parcial ou total, com exceção do cristalino, todos os tecidos do olho podem ser invadidos pelas microfilárias. As microfilárias podem causar manifestações cutâneas agudas, com prurido intenso, ou manifestações crônicas. O período de incubação da doença pode variar de sete meses a dois anos. A ocorrência da Oncocercose é mais comum em países da África Central e da América Central. No Brasil, a ocorrência da doença é mais comum na região Amazônica.

Durante a construção da Rodovia Transamazônica foi registrada outra doença desencadeada pelas picadas dos borrachudos, que ficou conhecida como "síndrome de Altamira" (nome da cidade onde foi feito o registro). A doença se manifesta pelo aparecimento de pequenas hemorragias, cobrindo toda a superfície do corpo ou restrita às faces ou às extremidades e, ainda; na mucosa bucal. Em 30\% dos casos foram observados sangramentos nas gengivas e na mucosa nasal.

A Mansonelose causada pela Mansonella ozzardi Manson, 1897 (CERQUEIRA, 1959; SHELLEY et al., 1980; MORAESet al., 1985) são duas graves enfermidades que podem ser transmitidas pelas picadas de vetores da família Simuliidae (MARDINI, 2002). Tem como sintomatologia dor nas pernas, articulações, febre, cefaleia, adenite inguinocrural, placas eritematopruginosas, eosinofilia entre outros.

Além dessas enfermidades de extrema importância em saúde pública, outras filárias, alguns vírus, bactérias e protozoários podem estar associados a esses insetos, mas necessitam de maiores estudos. Ainda outras doenças, como a síndrome hemorrágica de Altamira e o pênfigo 
foliáceo poderiam ter como determinantes as picadas desse inseto (GAONA e ANDRADE, 1999).

No Rio Grande do Sul, seu ataque às populações humanas provoca desde intenso desconforto aliado à necessidade de atendimento médico e a utilização de medicamentos em muitos casos, até a impossibilidade de desenvolver atividades cotidianas do trabalho na área rural. Secundariamente, causa o êxodo dos locais de grande incidência de ataque e redução do fluxo de turistas que procuram o conhecido turismo rural, turismo ecológico, atividades de trilhas, etc. (MARDINI et al., 1998; MARDINI et al., 2000).

Nas últimas décadas, foi publicado um número considerável de estudos ecológicos acerca da família Simuliidae nas regiões temperadas (CORKUM e CURRIE, 1987; CIBOROWSKI e ADLER, 1990; MCCREADIE e COLBO, 1991, 1992; MALMQVIST, 1994; MCCREADIE et al., 1995; WOTTON et al., 1998). No Brasil, a maioria dos poucos estudos recentes sobre o comportamento dos imaturos de borrachudos é restrita à Amazônia (HAMADA et al., 2002), à Mata Atlântica (PEPINELLI et al., 2005; FIGUEIRÓ et al., 2006, 2008; COPPO e LOPES, 2010; BERTAZO e FIGUEIRÓ, 2012) e ao Cerrado (LANDEIRO et al., 2009, FIGUEIRÓ et al., 2012; DOCILE et al., 2015).

Estudos como o de Alencar et al.,(2001) e McCreadieetal.,(2004) demonstram que os padrões distribucionais, bionômicos e comportamentais encontrados na região Neotropical podem ser muito diferentes dos padrões descritos para as regiões Neártica e Paleártica, o que aponta para a necessidade de maiores estudos nesta região para ajudar no controle efetivo.

Há escassez de estudos sobre os padrões de preferência de microhabitat dos borrachudos e estes padrões são conhecidos para poucas espécies neotropicais de Simuliidae (FIGUEIRÓ et al., 2012; FIGUERÓ e GIL-AZEVEDO, 2010).O pouco conhecimento dos padrões dificulta o controle desses insetos e a maioria dos métodos utilizados para controlar essas populações visa ao ataque às fases larvais por estas estarem fixas em microhabitat de ambientes lóticos. (SANTOS-JR et al., 2007; FIGUEIRÓ et al., 2008; BERTAZO e FIGUEIRÓ, 2012; FIGUEIRÓ et al., 2012).

Neste sentido, esse trabalho se propôs a identificar os estudos publicados na literatura que avaliaramas informações sobre as preferências de microhabitat da família Simuliidae (Diptera) na região Neotropical.

\section{Material e Métodos}

Os meios utilizados para obtenção dos dados referentes aos microhabitats dos simulídeos na região Neotropical foram Scielo, Pubmed, Sci-hub, Google Acadêmico, livros, revistas e artigos com um período de 1945 até 2015. A princípio as palavras-chaves utilizadas foram: simulídeos, região neotropical e microhabitat. E num segundo momento além das palavras citadas também foram utilizados os nomes dos países que compõem a região neotropical, bem como nomes das espécies encontradas em tais países.

Através do inventário taxonômico e geográfico mundial de simuliidae de (ADLER e CROSSKEY, 2015) foi feito o levantamento da diversidade das espécies da região Neotropical por países e pelos artigos os microhabitats relacionados às espécies.

\section{Resultados e Discussão}

Segundo GIL-AZEVEDO e MAIA-HERZOG (2009) as larvas dos Simuliidae são encontrados em microhabitat submersos de ambientes lóticos. O mais notável biologicamente nas larvas de simulídeos é o seu grande potencial colonizador, pois diversas espécies podem colonizar novos microhabitat em poucas horas (KIEL et al., 1998). 
Em suas conclusões LOZOVEI et al.,(2004) relatam que serrapilheira, algas e plantas aquáticas fornecem habitat adequado em córregos lentos para os simulídeos pois, estes desenvolveram adaptações para viver nesses ambientes.

Córregos que com fundo de areia, abundante vegetação marginal e plantas aquáticas retêm mais o produto à base de bacillus thuringiensis var. israelensis (Bti) para controle da população de simulídeos do que aqueles formados com fundo de pedra, e sem vegetação (TEIXEIRA, 2013).

Diversos pesquisadores utilizam fitilhos (microhabitat artificial) de cores variadas e verificam que estes são colonizados mais intensamente que os vegetais, pedras e cimento juntos (LOZOVEI et al., 2004).

Algumas espécies de vegetais também servem como microhabitat. De acordo com LUTZ (1922) apud (D'ANDRETTA e D'ANDRETTA JÚNIOR, 1945) as pupas de Simulium orbitale Lutz (1910) têm preferência em se fixar nas Podostemáceas, pois estas apresentam um papel central no fluxo de energia nos ecossistemas, sendo importantes na cadeia trófica dos ecossistemas aquáticos amazônicos (BOTERO et al., 2003; PETRY et al., 2003).

A região Neotropical compreende a área desde parte sul do México até o sul da América do Sul (GOLDANI, 2010), abrangendo 30 países que foram pesquisados. Segundo (GOLDANI e CARVALHO, 2003), a mesma é um ponto referencial importante no estudo dos padrões de distribuição dos seres vivos por apresentar uma biodiversidade alta e características climáticas e fisiográficas diversificadas. O Brasil é o país com mais estudos do microhabitat artificiais e naturais (Figuras 1 e 2) dos simulídeos entre os países da região Neotropical.

\section{Microhabitat Artificial de Imaturos no Brasil}

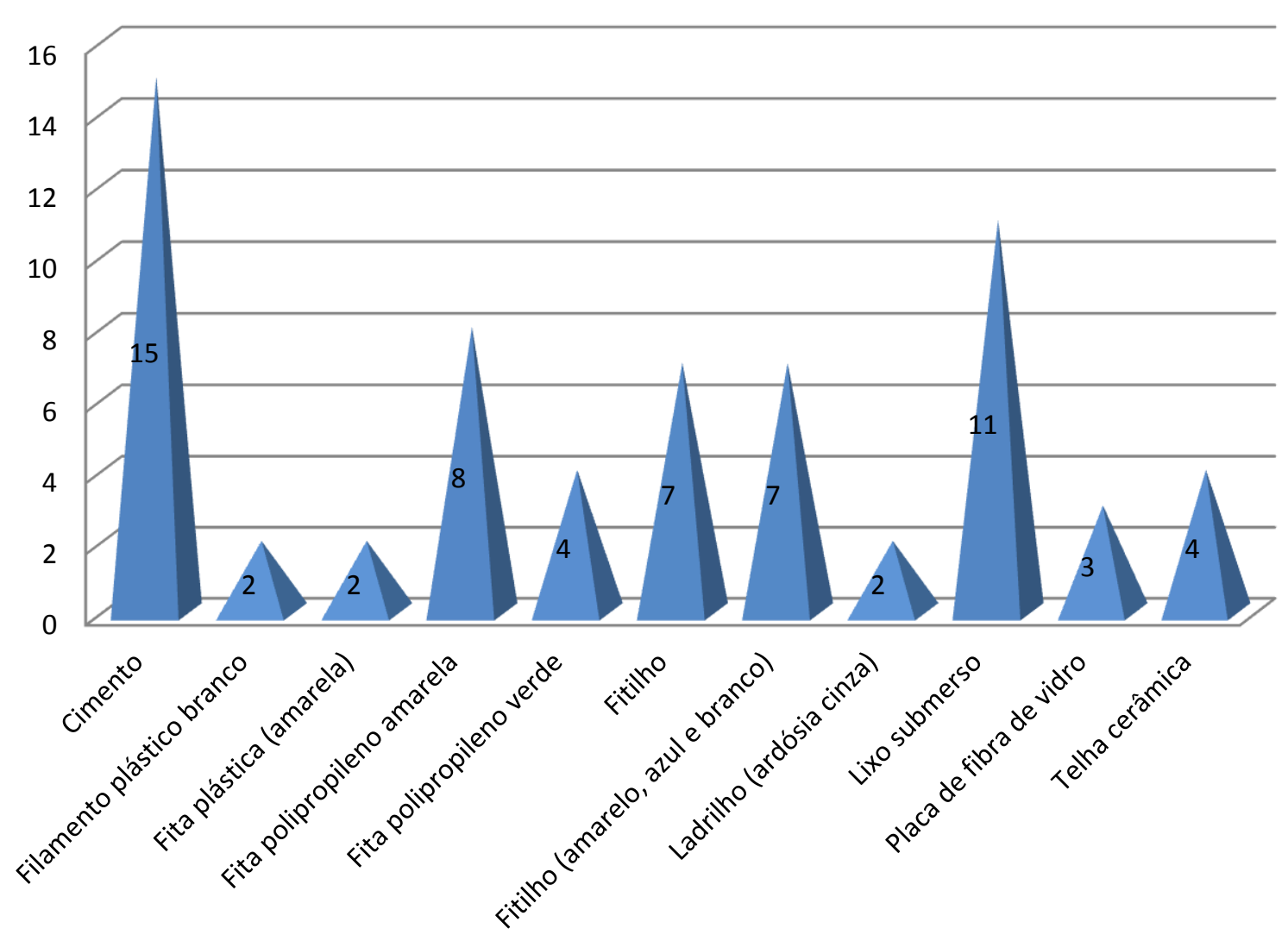

Figura 1: Microhabitat Artificial - quantidade de espécies descritas x microhabitat. 


\section{Microhabitat Natural de Imaturos no Brasil}

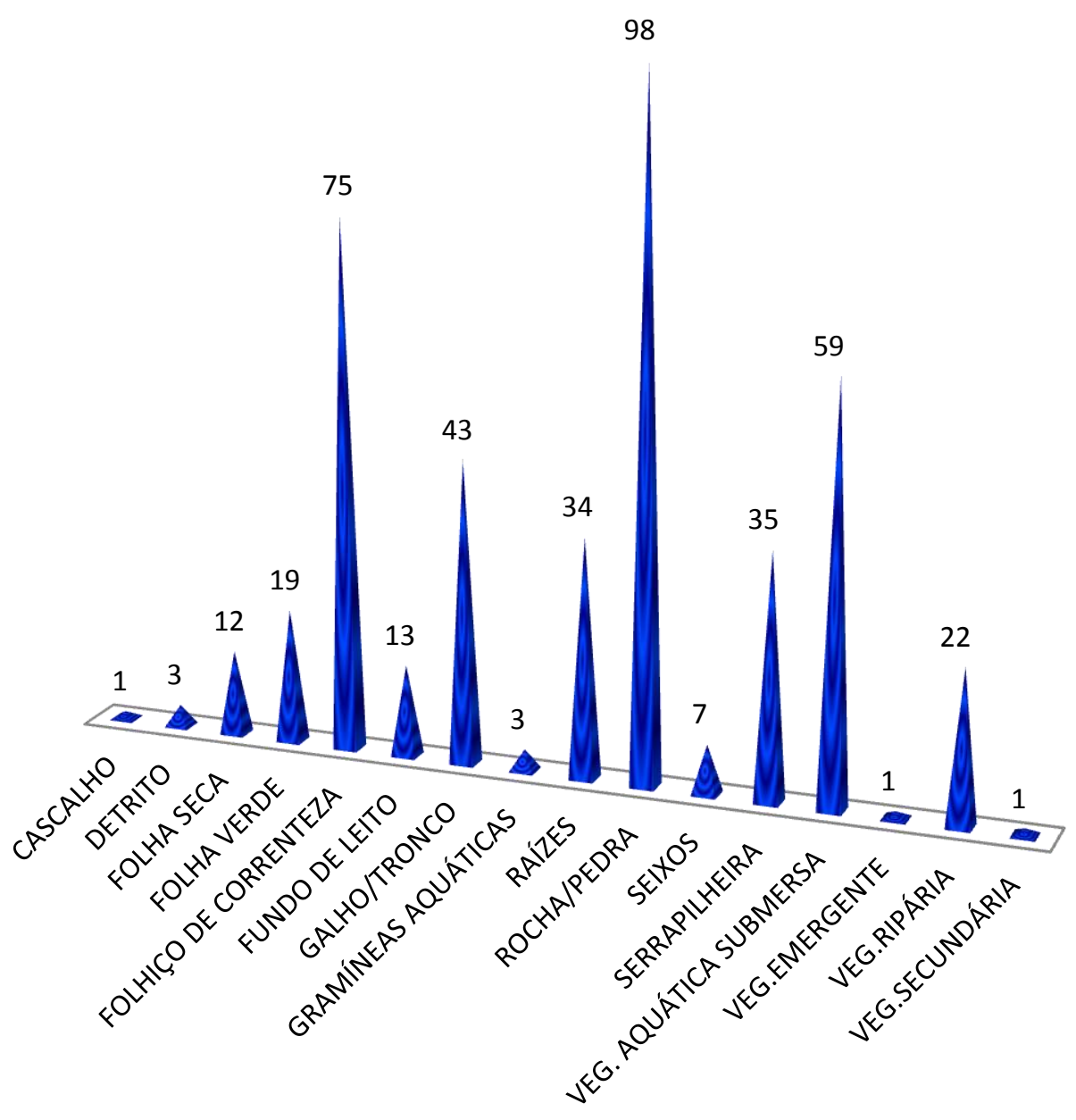

Figura 2: Microhabitat Natural - quantidade de espécies descritas x microhabitat.

Em sua pesquisa no Riacho dos Padres, município de Almirante Tamandaré, Curitiba, PR, Lozovei et al., (2004) fizeram levantamento das espécies de simulídeos e sua preferência de microhabitat naturais e artificiais naquela região.

Pode-se considerar que a espécie Simulium inaequale Paterson \& Shannon (1927) foi a que teve melhor desempenho e equilíbrio nos microhabitat com ocorrência em todos, enquanto o $S$. incrustatum Lutz (1910) ocorre esporadicamente (LOZOVEI et al., 2004).

Para Lozovei et al., (2004), provavelmente o desempenho da espécie S.inaequale ocorreu por adaptar-se melhor às condições ambientais e hidrológicas, onde a água enriquecida com nutrientes da ração de peixes propiciou um habitat adequado à procriação.

Na mesma pesquisa Lozovei et al, (2004) a abundância de larvas foi de um total de 89,17\% e as pupas somente $10,83 \%$. Os fitilhos utilizados foram mais colonizados com um percentual de $87,38 \%$ de larvas contra $12,62 \%$ em vegetais, pedras e cimento juntos.

$\mathrm{Na}$ análise da dinâmica populacional, há limitações na utilização de microhabitats artificiais porque podem diferir atrativamente para cada espécie (tamanho, cor, textura, forma, etc.), a qualidade pode mudar nas estações e o tempo de exposição (HAMADA et al., 1997). 
Ao utilizar filamentos de plásticos brancos, telhas (ardósia cinza) e fita amarela de plástico que ficaram sob a superfície da água, Hamada et al., (1997) verifica que o último microhabitat citado é o mais colonizado e que não houve diferença entre os outros dois microhabitats. Microhabitat artificial como, por exemplo, telhas cerâmicas, são mais adequadas para estudos de abundância sazonais devido à superfície lisa, durabilidade e fácil manuseio, segundo (LEWIS e BENNETT, 1974) apud (ARAUJO-COUTINHO et al., 1999).

A profundidade e tempo de exposição foram os principais fatores a afetar a colonização de larvas em um microhabitat, e a diminuição destas na corrente de fundo seria devido à proliferação de lodo no microhabitat do leito do rio (HAMADA et al., 1997) concluíram. A variação na ocorrência de espécies nos habitats de remanso ou correnteza pode estar associada às diferenças na velocidade do fluxo, quanto ao tipo, particulação e estabilidade dos microhabitat, como na qualidade dos alimentos disponíveis (EGLER, 2002).

Ao estudar as larvas de Simulium sp. no litoral norte de São Paulo (CUNHA, 2004) utilizou placas de fibra de vidro em diferentes pontos onde três espécies (S.pertinax, S.inaequale e S.incrustatum) colonizaram.

Simulium pertinax prefere microhabitat com maior oxigenação e menor nutrientes (nitrogênio amoniacal, nitrogênio total, fósforo total) e S.inaequale ao contrário e o S.incrustatum tolera microhabitat em níveis de poluição intermediária concluiu Cunha (2004).

Em sua pesquisa Figueiró et al., (2012) demonstraram que a maior parte das espécies de simulídeos nos rios do Cerrado (Tocantins, Brasil) têm uma tendência a associação com o folhiço de correnteza, exceto por Simulium nigrimanum Macquart (1838) à microhabitat rochoso, esta associação também foi descrita por (SHELLEY et al., 2002) e (FIGUEIRÓ et al., 2006). Enquanto Simulium cuasiexiguum Shelley, Luna Dias, Maia-Herzog \& Lowry (2000) tende a se associar à vegetação ripária provavelmente por ser o único microhabitat submerso e apropriado para imaturos de Simuliidae nos rios (FIGUEIRÓ et al., 2012).

Outra análise de (FIGUEIRÓ et al., 2012) foi tendência geral das larvas ocorrerem no meio dos rios, e não nas suas margens, com a exceção de Simulium nigrimanum e Simulium cuasiexiguum.

No Parque Nacional do Itatiaia FIGUEIRÓ (2004) identificou seis espécies de simulídeos: Simulium clavibranchium Lutz 1910, S.subnigrum, S.rappae Py-Daniel \& Coscarón (1982)quepertencem ao grupo Inaequale, compreendido no subgênero Inaequalium, S.incrustatum, Simulium sp e Lutzsimulium pernigrum.

Nas correlações entre as variáveis e a abundância de espécies (FIGUEIRÓ, 2004), cita que para Simulium sp. e Lutzsimulium pernigrum foram positiva com o microhabitat vegetal, enquantoo grupo Inaequale foi negativo.

Outra importante correlação de (FIGUEIRÓ, 2004) das espécies Simulium sp. e Lutzsimulium pernigrum é que no período de vazão mais elevada e de maior quantidade de microhabitat vegetal representado há ocorrências de ambas as espécies.

Em seu artigo (HAMADA, 1989) relacionou o microhabitat e a densidade das larvas de $S$. goeldii, e observou que eram mais abundantes em folha verde, mas sugere que a raiz e folha seca nos locais estudados sejam os mais importantes no estabelecimento desta espécie.

De acordo com (DELLOME FILHO, 1991) as espécies: S.incrustatum, S. pertinax, S.lewisi, S.subnigrum, S.orbitale, S.hirticosta, S.inaequale, Simulium riograndense Py-Daniel, Souza \& Caldas (1988) e seus imaturos viviam na face inferior do microhabitat, às vezes superpostos e não foram encontrados sobre rochas.

Com exceção de Simulium nigrimanum e Simulium cuasiexiguum a maioria das espécies demonstraram uma tendência para folhiços de correnteza associados com substrato rochoso e vegetação ripária, respectivamente, segundo Figueiró et al., (2012).

Os pesquisadores Coppo e Lopes (2010) identificaram 12 espécies em diferentes microhabitats. Simulium anamarie Vulcano (1962), S. riograndense e S. subclavibranchium 
foram capturados apenas em microhabitat rochoso, o que pode indicar preferência de colonização por este tipo de microhabitat.

A pesquisa de Benteset al., (2014) informou sobre o habitat (aquático/terrestre, igarapé/lago) e o tipo de microhabitat (fragmentos rochosos, folhiço, macrófitas enraizadas e flutuantes, raízes, galhos/troncos) de ovos/posturas de insetos aquáticos.

Em ambientes lóticos, os microhabitats submersos de ovos/posturas foram folhas de Tonina fluviatilis Aubl., Thurnia sp. e Ciperaceae, troncos, folhiço, raízes e fragmentos rochosos (BENTES et al.,2014).

Também foi observado (BENTES et al., 2014) ovos de Hemerodromia sp. (Empididae) em associação com ovos de S.perflavum e as fêmeas dessas duas famílias foram observadas ovipondo no mesmo local e ao mesmo tempo (HAMADA, 1993).

Uma questão levantada por (HAMADA et al., 2002) é que a maioria dos estudos sobre borrachudosneotropicais aproxima a ecologia da população de espécie individual (por exemplo, (HAMADA e McCREADIE, 1999), em vez de toda a comunidade de borrachudos. Nos últimos anos, alguns autores tentaram investigar a ecologia da comunidade de borrachudos (HAMADA et al.,2002; McCREADIE et al.,2004; PEPINELLI et al., 2005; FIGUEIRÓ et al.,2006, 2008, LANDEIRO et al., 2009), no entanto, as contribuições para este campo particular na região Neotropical da ecologia dos borrachudos ainda são muito limitadas.

Em suas análises Santos-Netoet al., (2015) observaram larvas S.guianense em diferentes microhabitat (galhos, ramos, macrófitas (Podostemaceae) e rochas (com alta densidade de larvas e pupas), que não é um microhabitat comum para a espécie. (GOMES e PY-DANIEL, 2002 apud Santos-Netoet al., (2015) atribuíram o uso de pedras e raízes submersas como microhabitat dessa espécie às mudanças ambientais devido à construção de uma barragem no estado do Amazonas, o que quase provocou a extinção de Podostemaceae em sua área de estudo.

Ao analisar a preferência das larvas de S.goeldii por algum tipo de microhabitat estima a quantidade de larvas e a disponibilidade de cada microhabitat na área de amostragem, onde observou uma quantidade numerosa das larvas em folhas verdes, porém esse microhabitat não era tão disponível quanto na raiz e folhas secas sendo estes últimos mais relevantes na colonização dessa espécie (HAMADA, 1989)

No Riacho dos Padres Petry et al. (2004), citam que a laje de cimento do vertedouro, é microhabitat ideal à procriação de simulídeos por produzir alto teor de oxigênio dissolvido na água, bem como a vegetação aquática nas margens do riacho ou parcialmente submersa.

Foram encontradas sete espécies de simulídeos(S.inaequale, S.perflavum, S.pertinax, S.orbitale, S.subnigrum, S. distinctum, S.incrustatum), onde tais colonizaram os mesmos microhabitats vegetais, galhos, folhas caídas e represadas, plantas aquáticas e semiaquáticas e artefatos de lixo doméstico e seus percentuais de ocorrência foram (PETRY et al., 2004).

Essas espécies colonizaram os mesmos microhabitat, mas as três primeiras se destacaram por frequência constante em todos os microhabitat. Enquanto as outras colonizaram os microhabitat em número reduzido de exemplares e em freqüência irregular (PETRY et al.,2004).

Nos córregos do Parque Estadual Intervales, SP (PEPINELLI et al.,2005) identificaram as espécies de Simuliidae presentes e caracterizaram os seus microhabitat. Nos seis córregos analisados foram encontradas 13 espécies de simulídeos em seu estágio de larva, pupa e ovo e seus microhabitats (folhas, galhos e seixos). Em seguida, tiveram como conclusão que em cinco córregos presente no estudo, as espécies S.nogueirai e S.subclavibranchium foram mais numerosos em microhabitat de folhas e galhos no leito do córrego e plantas marginais.S. incrustatum Lutz, S.subnigrum Lutz e S.inaequale Paterson e Shannon foram coletados em apenas dois córregos, sendo o primeiro apenas encontrado sobre folhas e galhos em decomposição e outros dois mais encontrados em ambientes como maior incidência de luz.

Nas margens do Rio das Mortes (PEPINELLI et al., 2005), que tem águas turbulentas em relação aos outros córregos foram amostrados imaturos de S.lutzianum Pinto sobre ramos e 
folhas de vegetação caídas. Enquanto, na Bica do Mirante, as larvas de S.dinelli foram encontradas fixadas a musgos. Em um dos coletores nas proximidades desse rio encontrou o único exemplar de S. Psaroniocompsa sp. (fêmea) em atividade hematofágica, possivelmente pertencente ao complexo S.oyapockense do grupo amazonicum (CROSSKEY e HOWARD, 1997 apud (PEPINELLI et al., 2005).

O efeito da poluição sobre a distribuição de borrachudos e a distribuição do microhabitat, é pouco estudado na região Neotropical e com esse objetivo (PINTO et al., 2014) investigaram a associação das espécies a diferentes graus de integridade ambientais e fatores abióticos.

Um dos fatores ambientais que podem causar explosões populacionais em borrachudos é o aumento da entrada de matéria orgânica em cursos de água, devido a lixo doméstico é o que relata (PINTO et al., 2014).

Simulium (Chirostilbia) spp. e S.incrustatum estão associados a velocidade de água lenta e a degradação do habitat e S.subnigrum à correntes mais rápidas e ser indicador de integridade do habitat (PINTO et al., 2014).

Em Cudinamarca na Colômbia Arteaga eDe Hoyos (1999) encontraram S. metallicum em riachos e rios que fluem lentamente aderido a folhas verdes e gramíneas submersas como correlaciona (GRILLET et al., 1995), sacos plásticos ou garrafas serviram como microhabitat artificial, mas raramente foram encontrados em rochas.

As larvas foram mais abundantes em rochas submersas e folhas caídas, embora os padrões de consumo no microhabitat revelem partilhas dos mesmos entre a maioria das espécies de borrachudos, segundo GRILLET e BARRERA (1997).

Analisando os microhabitat (pedra, folhas, raízes e galhos) das espécies S.ochraceum e S.paynei (GRILLET \& BARRERA, 1997) observaram a preferência destas pelas raízes. Em um córrego lento de $1 \mathrm{~m}$ de largura e $10 \mathrm{~cm}$ de profundidade SHELLEY et al.,(2002) coletaram S.ochraceum ligado a folhas caídas. Enquanto GRILLET e BARRERA (1997) a encontrou em cada microhabitat, mas utilizando raízes comparativamente mais submersas do que a maioria de espécies.

As larvas de $S$. metallicum e Simulium sp. estavam ligadas a folhas e galhos, e as raízes e folhas, respectivamente, em uma proporção maior do que o esperado para a aliança (GRILLET e BARRERA, 1997).

As larvas foram mais abundantes em rochas submersas (49\%) durante a estação chuvosa e folhas caídas (47\%) nos meses de menor precipitação, em pequenas áreas rasas de corrente de água lenta, alta condutividade e esparsa cobertura vegetal terrestre (GRILLET et al., 1995).

Simulium metallicum é uma espécie generalista em sua fase larval por ocuparem dois microhabitat (rochas e folhas mortas) sugerem (GRILLET et al., 1995). Em sua pesquisa SHELLEY et al. (2002) coletaram S.metallicum em córregos lentos de larguras e profundidades variadas e sempre ligados a folhas caídas, gramíneas e vegetação submersa.

A espécie $S$. guianense é o principal vetor nas áreas de montanhosas, planaltos altos ou áreas de cerrado do foco amazônico, tanto no Brasil como no sul da Venezuela (GRILLET et al., 2001 e VILLAMIZAR et al., 2011). Ao descreverem o primeiro habitat de S. guianense, corroborou o fato de que as fêmeas de $S$. guianense fazem oviposição especificamente em áreas de corredeiras rápidas e grandes rios turbulentos e cachoeiras e que a espécie é "especialista" (VILLAMIZAR et al., 2011).Os estágios imaturos do S.guianense foram coletados em plantas aquáticas (Podostemaceae) VILLAMIZAR et al., (2011).

Imaturos da espécie S.samboni foram coletados ligados a folhas, gramíneas e vegetação submersa no centro sul da Montanha Pine Ridge numa altitude entre 400 - $700 \mathrm{~m}$ e entre 40 400 m ligadas a folhas caídas e vegetação emergente, no sul de Belize (SHELLEY et al., 2002).

Simulium cormonsi Wygodzinsky (1971) em seu microhabitat é comparada por (MONCADA et al., 2013), onde as mesmas foram encontradas no Parque Nacional Chingaza na Colômbia ligadas às pedras e vegetação ripária: tais como as folhas de bambu montanha 
(Chusquea) enquanto na Venezuela, larvas e pupas foram encontrada em vegetação ripária e superfícies superior e inferior de pedras caídas em córregos rochosos, 3-5 m de largura (WYGODZINSKY, 1971). A espécie Simulium muiscorum Bueno, Moncada \& Muñoz de Hoyos (1979) também foi coletada sobre rocha e vegetação ripária no Parque Nacional Chingaza (SALCEDO, 2014).

No lago Otún no alto dos Andes colombianos MANTILLA et al., (2013) coletaram duas novas espécies Simulium quimbayium Mantilla, Moncada, Matta \& Adler (2013) em rochas e vegetação ripária em número muito maior do que Simulium machetorumMantilla, Moncada, Matta \& Adler (2013) que colonizando vegetação ripária foi encontrado em número muito reduzido.

O S.oyapockense está presente na Bolívia, Brasil, Colômbia e Venezuela e picos populacionais ocorrem na estação de chuvas, fazendo que rios grandes ou pequenos inundem seus bancos e forneça vegetação submersa para reprodução dessa espécie, segundo (SHELLEY, 1988).

\section{Panorama e perspectivas para o controle}

As primeiras tentativas de controle de simulídeos no Brasil tiveram início em 1957, através da aplicação de organoclorados de forma aleatória (SUCEN 1977). Entretanto, devido à ação residual associada a estes larvicidas, os organoclorados foram gradualmente substituídos pelos organofosforados na década de 70 do séc. XX, substâncias que embora menos agressivas ao ambiente ainda afetavam a entomofauna associada (Ruas-Neto, 1984).

O tempo de exposição é um fator que diferencia a forma de aplicação de larvicidas para simulídeos da aplicação para mosquitos, pois em condições naturais o tempo de exposição das larvas ao inseticida é breve e diretamente relacionado às condições hidrológicas do rio (Lacey, 1997). Devido a este fator, o conhecimento das condições hidrológicas dos microhabitats de simulídeos pode nortear a aplicação de larvicidas aos criadouros naturais preferenciais destes organismos, assim otimizando sua eficiência e reduzindo impactos ambientais. Portanto, para o entendimento da dinâmica de transmissão e controle da doença é fundamental o conhecimento dos criadouros desses vetores.

\section{Referências}

ADLER, P. H.; CROSSKEY, R. W. World Blackflies (Diptera: Simuliidae): A Comprehensive Revision of the Taxonomic and Geographical Inventory [2015], 123 pp. South Carolina, Clemson University.

ALENCAR, Y.B.; LUDWIG, T.A.V.; SOARES, C.C.; HAMADA, N. Stomach Content Analyses of Simulium perflavum Roubaud 1906 (Diptera: Simuliidae) Larvae from Streams in Central Amazônia, Brazil. Mem. Inst. Oswaldo Cruz, Rio de Janeiro, v. 96, n. 4, p. 561-576, 2001.

ARAÚJO-COUTINHO, C.J.P.C.; MELLO, R. P.; FREIRE, N. M. S. The seasonal abundance of Simulim (Chirostilbia) pertinax, Kollar 1832,(Diptera, Simuliidae) and related entomological fauna in the municipality of Paraty, RJ, Brasil. Revista Universidade Rural, série ciênc. vida, v. 21, p. 107-116, 1999.

ARTEAGA, L.T.; DE HOYOS, P. M.. New cytotype in the Simulium metallicum complex (Diptera: Simuliidae) from Cundinamarca, Colombia. Journal of medical entomology, v. 36, n. 2, p. 133-140, 1999. 
BENTES, S.P.C.; HAMADA, N.; FERREIRA-KEPLER. R.L. Caracterização morfológica de ovos de insetos aquáticos e seus habitats na Amazônia central, Brasil. INPA - Instituto Nacional de Pesquisa da Amazônia. (Org.) Insetos Aquáticos na Amazônia brasileira: taxonomia, biologia e ecologia. 1 ed. Manaus: INPA, 2014. Cap. 4, p. $52-68$.

BERTAZO, K.; FIGUEIRÓ, R. Spatial distribution of black fly (Diptera: Simuliidae) immatures in a water current velocity gradient in Aracruz/ES, Brazil. Revista de Ciências da Vida, v. 32, n. 2, p. 91-101, 2012.

BOTERO, J. I. S.; FARIAS, M. L.; PIEDADE, M. T., GARCEZ, D. S. Ictiofauna associada às macrófitas aquáticas Eichhornia azurea (SW.) Kunth. e Eichhornia crassipes (Mart.) Solms. no lago Camaleão, Amazônia Central, Brasil-DOI: 10.4025/actascibiolsci. v.25i2. 2026. Acta Scientiarum. Biological Sciences, v. 25, n. 2, p. 369-375, 2003.

CAMPOS, J. G.; ANDRADE, C.F.S. Aspectos da Entomologia Médica e Veterinária dos borrachudos (Díptera, Simuliidae) - Biologia, Importância e Controle. LECTA, v.17, p.51-65, 1999.

CERQUEIRA, N.L. 1959. Sobre a transmissão da Mansonella ozzardi $1^{\mathrm{a}}$ e $2^{\mathrm{a}}$ notas (Trabalho do Inpa). Jornal Brasileiro de Medicina, I(7): 885-914.

CIBOROWSKI, J.J.; P.H. ADLER. Ecological segregation of larval black flies (Diptera: Simuliidae) in northern Saskatchewan, Canada. Can. J. Zool. 68: 2113-2122, 1990

COPPO, T. L.; LOPES, J. Diversidade de Simuliidae (Diptera: Nematocera) de três cursos d'água no parque ecológico da Klabin SA-Telêmaco Borba, Estado do Paraná. Semina: Ciências Biológicas e da Saúde, v. 31, n. 1, p. 03-14, 2010.

CORKUM L D, CURRIE D. C. Distributional of immature Simuliidae (Diptera) in northwestern North America. Fresh Biol 17: 201-221, 1987.

COUCEIRO, M.E., LÓPEZ, L.B., ÁVILA BLAS, O.J. Distribución del bajo peso para la edad gestacional en el municipio capital de la provincia de Salta. República Argentina. Antropo, 32, 55-67, 2014.

CROSSKEY, R. W. The Natural History of Blackflies. New York: John Wiley \& Sons, 1990. $711 \mathrm{p}$.

CUNHA, A.B.P.V. Variação temporal e sazonal de larvas de Simulium sp. No litoral norte do estado de São Paulo. 2004. 58f. Dissertação (Mestrado em Aquicultura). Área de concentração: Aquicultura em Águas Continentais - Universidade Estadual Paulista, São Paulo.

D'ANDRETTA, M. A.V.; D'ANDRETTA JÚNIOR, C. As espécies neotropicais da família Simuliidae Schiner, 1864 (Diptera-Nematocera): I. Simulim (Eusimulium) orbitale Lutz, 1910, Simulium (Eusimulium) pintoi $n$. sp. e Simulium nigrimanum MACQUART, 1837, sp. inquirendae. Memórias do Instituto Oswaldo Cruz, v. 43, n. 1, p. 85-152, 1945. 
DELLOME FILHO, J. Simuliofauna do Rio Marumbi (Morrete, Paraná, Brasil). I Coleta e criação; Dados meteorológicos e físico-químicos do criadouro; adultos (Diptera, Simuliidae. Acta Biológica Paranaense, v. 20 n. 1, 2, 3, 4, p. 145-156, 1991.

DOCILE, T.N., FIGUEIRO, R., AZEVEDO, H.G. \& NESSIMIAN, J.L. (2015) Water pollution and distribution of the blackfly (Diptera: Simuliidae) in the Atlantic Forest, Brazil. Revista de Biologia Tropical, v. 63, p. 683-693.

EATON, D.P.; DIAZ, L.A.; HANS-FILHO, G.; SANTOS, V. DOS; AOKI, V.; FRIEDMAN, H.; RIVITTI, E.A.; SAMPAIO, S.A.P.; GOTTLIEB, M.S.; GIUDICE, G.J.; LOPEZ, A.; CUPP, E.W. Comparison of black fly species (Diptera: Simuliidae) on an amerindian reservation with a high prevalence of fogo selvagem to neighboring disease-free sites in the state of Mato Grosso do Sul, Brazil. Journal of medical entomology, v. 35, n. 2, p. 120-131, 1998.

EGLER, M. Utilizando a Comunidade de Macroinvertebrados Bentônicos na Avaliação da Degradação de Ecossistemas de Rios em Áreas Agrícolas. 2002. 166f. Dissertação (Mestrado em Ciência). Área de concentração: Saúde Pública - Fundação Oswaldo Cruz, Rio de Janeiro.

FIGUEIRÓ, R. Simulídeos (Diptera: Simuliidae) do Parque Nacional do Itatiaia RJ/MG, Brasil. 2004. 90f. Dissertação (Mestrado em Ciências Biológicas). Área de Concentração: Ecologia - Universidade Federal do Rio de Janeiro, Rio de Janeiro.

FIGUEIRÓ, R.; ARAÚJO-COUTINHO; C.J. P. C., GIL-AZEVEDO; L.H., NASCIMENTO; E.S., \& MONTEIRO, R.F. (Spatial and temporal distribution of blackflies (Diptera: Simuliidae) in the Itatiaia National Park, Brazil. Neotropical Entomology, v. 35, n. 4, 542-550, 2006.

FIGUEIRÓ, R.; NASCIMENTO, É.S.D.; GIL-AZEVEDO, L. H., MAIA-HERZOG, M., \& MONTEIRO, R. F. Local distribution of blackfly (Diptera, Simuliidae) larvae in two adjacent streams: the role of water current velocity in the diversity of blackfly larvae. Revista Brasileira de Entomologia, v.52, n. 3, 452-454, 2008.

FIGUEIRÓ, R.; GIL-AZEVEDO, L.H. The role of Neotropical blackflies (Diptera: Simuliidae) as vectors of the onchocerciasis: A short overview of the ecology behind the disease. Oecologia Australis, v. 14, n. 3, p. 745-755, 2010.

FIGUEIRÓ, R.; GIL-AZEVEDO, L.H.; MAIA-HERZOG, M.; MONTEIRO, R.F. Diversity and microdistribution of black fly (Diptera: Simuliidae) assemblages in the tropical savanna streams of the Brazilian cerrado. Memórias do Instituto Oswaldo Cruz, v. 107, n. 3, p. 362-369, 2012.

GAONA, J. C.;ANDRADE, C. F. S. Aspectos da entomologia médica e veterinária dos borrachudos (Diptera:Simuliidae) - Biologia, Importância e controle. LECTA, São Paulo, v.17, n.1, p.51-65, 1999.

GIL-AZEVEDO, L.H.; MAIA-HERZOG, M. Espécies de Simuliidae (Diptera) registradas no Estado do Rio de Janeiro, Brasil. Arquivos do Museu Nacional, v. 67, n. 3-4, p. 349-354, 2009.

GOLDANI, Â.; CARVALHO, G. S. Análise de parcimônia de endemismo de cercopídeos neotropicais (Hemiptera, Cercopidae). Revista Brasileira de Entomologia, v. 47, n. 3, p. 437 $442,2003$. 
GOLDANI, Â. Aplicabilidades e estudo comparativo da biogeografia histórica na região neotropical como ferramentas para conservação: os métodos" análise de parcimônia de endemismo" e "panbiogeografia". 2010. 122f. Tese (Doutorado em Zoologia) - Pontifícia Universidade Católica do Rio Grande do Sul, Rio Grande do Sul.

GRILLET, M. E.; BARRERA, R.; CONN, J. Simulium metallicum cytospecies E larval habitat characterization in the Altamira focus of onchocerciasis, northern Venezuela. Medical and veterinary entomology, v. 9, n. 2, p. 195-201, 1995.

GRILLET, M.E.; BARRERA, R. Spatial and temporal abundance, substrate partitioning and species co-occurrence in a guild of Neotropical blackflies (Diptera: Simuliidae). Hydrobiologia, v. 345, n. 2-3, p. 197-208, 1997.

GRILLET, M.E; BASANEZ M.G.; VIVAS-MARTINEZ, S.; VILLAMIZAR, N.; FRONTADO, H.; CORTEZ, J.; CORONEL, P.; BOTTO, C. Human onchocerciasis in the Amazonian area of southern Venezuela: spatial and temporal variations in biting and parity rates of black fly (Diptera: Simuliidae) vectors. Journal of Medical Entomology, v. 38, n. 4, p. 520-530, 2001.

HAMADA, N. Aspectos Ecológicos Simulium goeldii (Diptera: Simuliidade) relação entre substrato e densidade de larvas. Memórias do Instituto Oswaldo Cruz, Rio de Janeiro, v. 84, n. 4, p. 263-266, 1989.

HAMADA, N. Association between Hemerodromia sp. (Diptera, Empididae) and Simulium perflavum (Diptera, Simuliidae) in Central Amazonia, Brazil. Memórias do Instituto Oswaldo Cruz, v. 88, n. 1, p. 169-170, 1993.

HAMADA, N.; COSTA, W.L.S.; DARWICH, S.M. Notes on artificial substrates for black fly (Diptera: Simuliidae) larvae and microsporidian infection in central Amazonia, Brazil. Anais da Sociedade Entomológica do Brasil, v. 26, n. 3, p. 589-593, 1997.

HAMADA, N.; McCREADIE, J. Environmental factors associated with the distribution of Simulium perflavum (Diptera: Simuliidae) among streams in Brazilian Amazonia Hydrobiologia, v. 397, p.71-78, 1999.

HAMADA, N.; MCCREADIE, J.W.; ADLER, P.H. Species richness and spatial distribution of blackflies (Diptera: Simuliidae) in streams of Central Amazonia, Brazil. Freshwater Biology, v. 47, n. 1, p. 31-40, 2002.

KIEL, E.; BÖGE, F.; RÜHM, W. Sustained effects of larval blackfly settlement on further substrate colonisers. Hydrobiologie, v. 141, n. 2, p. 153-166, 1998.

LANDEIRO, V.L.; PEPINELLI, M.; HAMADA, N. Species richness and distribution of blackflies (Diptera: Simuliidae) in the Chapada Diamantina region, Bahia, Brazil. Neotropical entomology, v. 38, n. 3, p. 332-339, 2009.

LOZOVEI, A. L. PETRY, F.; NETO, L.G.S; FERRAZ, M.E.. Survey of the Simulium species (Diptera, Simuliidae), Riacho dos Padres, Almirante Tamandaré municipality, Paraná, Brazil. Revista Brasileira de Entomologia, v. 48, n. 1, p. 91-94, 2004.

MALMQVIST, B. Preimaginal blackflies (Diptera: Simuliidae) and their predators in a central Scandinavian Lake outlet stream. Annales Zoologici Fennici 31: 245-255, 1994. 
MANTILLA, J. S.; MONCADA, L. I.; MATTA, N. E.; ADLER, P. H. Two new species of black flies (Diptera: Simuliidae) from the High Andes of Colombia. Zootaxa, v. 3700, n. 3, p. 423-434, 2013.

MARDINI, L. B. L. F.; SOUZA, M .A. T.; GERALDO, L. S.; ATZ, A. M. V.. I FÓRUM GEOBIO- HIDROLOGIA. Medidores fixos de vazão em pequenas bacias rurais para utilização de larvicida biológico no controle de Simulium sp (DIPTERA - SIMULLIDAE) no Rio Grande do Sul, Brasil. 1998 p.184-189.

MARDINI, L. B. L. F.; SOUZA, M .A. T.; GERALDO, L. S.; ATZ, A. M. V. Simulium control Program. In: Rio Grande do Sul. Mem. Inst. Oswaldo Cruz, v.95, n.1, p.211-214, 2000. Suplemento.

MARDINI, Lucia Beatriz Lopes Ferreira; WILSON, Jorge Sebastião; TORRES, Maria Amélia ; ABBAD, Paulo Renato ; OLIVEIRA, Salzano Barreto de; MENEZES, Valter Jandir Vasconcellos . Simulídeos - Programa Estadual - Guia para Orientação aos Municípios sobre Manejo Integrado, Controle e Gestão de Insetos da Família Simuliidae (Diptera, Nematocera) no Rio Grande do Sul.Porto Alegre: CEVS, 2006. v. 1. 41p.

MCCREADIE, J. e M.H. COLBO. Spatial distribution patterns of larval cytotypes of the Simulium venustum / verecundum complex on the Avalon Peninsula, Newfoundland: Factors associated with occurrence. Can. J. Zool. 69: 2651-2659, 1991.

MCCREADIE, J. e M.H. COLBO. Spatial distribution patterns of larval cytotypes of the Simulium nenustum / verecundum complex on the Avalon Peninsula, Newfoundland: Factors associated with cytotype abundance and composition. Can. J. Zool. 70: 1389-1396. 1992

MCCREADIE, J., P.H. ADLER; M.H. COLBO. Community structure of larval black flies (Diptera: Simuliidae) from the Avalon Peninsula, Newfoundland. Ann. Entomol. Soc. Am. 88: 51-57, 1995.

MCCREADIE, J.W.; HAMADA, N.; GRILLET, M.E. Spatial-temporal distribution of preimaginal blackflies in Neotropical streams. Hydrobiologia, v. 513, n. 1-3, p. 183-196, 2004.

MONCADA L.A.; MANTILLA, S.; LOTTA, I.; MATTA, N.; ADLER, P.H. Simulium cormonsi Wygodzinsky (Diptera: Simuliidae) in Colombia: First record, egg and larval descriptions, and bionomics. Entomological Society of Washington, v. 115, n. 1, p. 103-106, 2013.

MORAES, M.A.P.; Shelley, A.J.; Luna Dias, A.P.A. 1985. Mansonella ozzardi no Território Federal de Roraima. Distribuição e achado de um novo vetor na área do rio Surumu. Memórias do Instituto Oswaldo Cruz, 80(4): 395-400.

PEDROSO-DE-PAIVA, D.;BRANCO,E.P. O BORRACHUDO NOCÕES BÁSICAS DE BIOLOGIA E CONTROLE.2000.Disponível em: http://www.infoteca.cnptia.embrapa.br/bitstream/doc/438645/1/CUsersPiazzonDocumentsPronto SCNPSADOCUMENTOS23OBORRACHUDONOCOESBASICASDEBIOLOGIAECONTRO LEFL20763A.pdf. Acesso em: 26 set. 2014. 
PEPINELLI, M.; TRIVINHO-STRIXINO, S.; HAMADA, N. Imaturos de Simuliidae (Diptera, Nematocera) e caracterização de seus criadouros no Parque Estadual Intervales, SP, Brasil. Revista Brasileira de Entomologia, v. 49, p. 527-530, 2005.

PETRY, F.; LOZOVEI, A.L.; FERRAZ, M.E.; SANTOS-NETO, L.G. Controle integrado de espécies de Simulium (Diptera, Simuliidae) por Bacillus thuringiensis e manejos mecânicos no riacho e nos vertedouros de tanques de piscicultura, Almirante Tamandaré, Paraná, Brasil. Revista Brasileira de Entomologia, v. 48, n. 1, p. 127-132, 2004.

PETRY, P.; BAYLEY, P.B.; MARKLE, D.F. Relationships between fish assemblages, macrophytes and environmental gradients in the Amazon River floodplain. Journal of Fish Biology, v. 63, n. 3, p. 547-579, 2003.

PINTO, M.J.R.; LUCENA, L.B.; MAIA, A.; DOCILE, T.N.; FIGUEIRÓ, R. A preliminary report on the microdistribution of black fly (Diptera:Simuliidae) larvae from the Serra dos Órgãos region, Brazil, and its habitat quality traits. Acta Scientiae \& Technicae, v. 2, n. 2, 2014.

REY, L., 1991. Parasitologia, Ed. Guanabara-Koogan, 2a ed.

RUAS-NETO, A.L. Bacillus thuringiensis var. israelensis como alternativa de controle de simulídeos no RioGrande do Sul. I. Susceptibilidade a campo. Boletim Saúde11: 21-26, 1984.

SANTOS-JR, J. E.; STRIEDER, M. N.; FIORENTIN, G. L.; NEISS, U. G.. Velocidade da água e a distribuição de larvas e pupas de Chirostilbia pertinax (Kollar, 1832)(Diptera: Simuliidae) e macroinvertebrados associados. Revista Brasileira de Entomologia, v. 51, p. 62-66, 2007.

SALCEDO, A.S.D. Taxonomia integrativa de la especie Simulium (Trichodagmia) muiscorum Bueno, Moncada \& muñoz de Hoyos, 1979 (Díptera: simuliidae) Presente en el Parque Nacional Natural Chingaza. Tese de Doutorado. Universidad Nacional de Colombia, 2014.

SANTOS-NETO, C. R.; HAMADA, N.; COUCEIRO, S.R.M. Bionomics of the black fly Simulium guianense (Diptera: Simuliidae) in northeast Brazil. Florida Entomologist, v. 98, n. 2, p. 446-450, 2015.

SHELLEY, A.J.; Luna Dias, A.P.A.; Moraes, M.A.P. 1980. Simulium species of the amazonicum group as vectors de Mansonella ozzardi in the Brazilian Amazon. Trans Royal Society of Tropical Medicine Hygiene, 74: 784-788.

SHELLEY, A. J. Biosystematics and distribution of Simuliid vectors of human onchocerciasis in South America. Memorias do Instituto Oswaldo Cruz, v. 83, n. 4, p. 399-403, 1988.

SHELlEY, A. J.; HERNÁNDEZ, L. M.; PENN, Malcolm. A biosystemic revision of the blackflies (Diptera: Simuliidae) of Belize, Central America. Bulletin of the Natural History Museum: Entomology, v. 71, n. 02, p. 135-271, 2002.

SUCEN. Superintendência de controle de endemias. Relatório das atividades do programa de controle desimulídeos. São Paulo. Editora Sucen. 81p, 1977. 
TAUIL, Pedro Luiz. Controle de doenças transmitidas por vetores no sistema único de saúde. Inf. Epidemiol. Sus, Brasília , v. 11, n. 2, p. 59-60, jun. 2002.

TEIXEIRA, T.Z. Avaliação da susceptibilidade e do controle de borrachudos (Diptera:Simuliidae) pelo emprego de larvicidas à base de Bacillus Thuringiensis var.israelensis (Bti) e Temepho. 2013. 82f. Dissertação (Mestrado em Ciência e Tecnologia Ambiental). Área de Concentração: Tecnologia Ambiental - Universidade Federal da Grande Dourados, Mato Grosso do Sul.

VILLAMIZAR, N.; CORTEZ, J.; ALARACÓN, O.N.; ESCALONA, M.; BOTTO, C.; GRILLET, M.E. First description of the Simulium guianense sl larval habitat in the Amazonian focus of onchocerciasis, southern Venezuela. Boletín de Malariología y Salud Ambiental, v. 51, n. 1, p. 97-101, 2011.

WOTTON, R.S., MALMQVIST, B., MUOTKA, T., LARSSON, K.. Fecal pellets from a dense aggregation of suspension-feeders in a stream: an example of ecosystem engineering. Limnol. Oceanogr. 43, 719-725, 1998.

WYGODZINSKY, P.W. Descriptions and redescriptions of species of the blackfly genus Simulium from the northern Andes (Simuliidae, Diptera). American Museum novitates; no. 2447, 1971. 